\title{
One-dimensional dispersion phenomena in terms of fractional media
}

\author{
W. Sumelka ${ }^{1, a}$, R. Zaera ${ }^{2, b}$, and J. Fernández-Sáez ${ }^{2, c}$ \\ 1 Poznań University of Technology, Institute of Structural Engineering, Piotrowo 5 street, 60-969 Poznań, Poland \\ 2 Department of Continuum Mechanics and Structural Analysis, University Carlos III of Madrid, 28911 Leganés, Madrid, Spain
}

Received: 27 April 2016 / Revised: 26 July 2016

Published online: 19 September 2016

(C) The Author(s) 2016. This article is published with open access at Springerlink.com

\begin{abstract}
It is well know that structured solids present dispersive behaviour which cannot be captured by the classical continuum mechanics theories. A canonical problem in which this can be seen is the wave propagation in the Born-Von Karman lattice. In this paper the dispersive effects in a 1D structured solid is analysed using the Fractional Continuum Mechanics (FCM) approach previously proposed by Sumelka (2013). The formulation uses the Riesz-Caputo (RC) fractional derivative and introduces two phenomenological/material parameters: 1 ) the size of non-local surrounding $l_{f}$, which plays the role of the lattice spacing; and 2) the order of fractional continua $\alpha$, which can be devised as a fitting parameter. The results obtained with this approach have been compared with the reference dispersion curve of Born-Von Karman lattice, and the capability of the fractional model to capture the size effects present in the dynamic behaviour of discrete systems has been proved.
\end{abstract}

\section{Introduction}

A key characteristic of the formalism based on the Classical Continuum Mechanics (CCM) is that its governing equations lack of an internal length and, for this reason, it is not fully applicable when effects like size-dependency and scaling of mechanical phenomena play a crucial role. A canonical problem in which this can be seen (crucial in herein considerations) is the wave propagation in an infinite discrete chain composed by concentrated mass linked by linear spring - the well-known Born-Von Karman lattice [1]. From the solution of this model it is clear that the wave propagation is dispersive in nature, in contrast to the 1D classical continuum approach which predicts that the velocity of propagation is independent of the wave number.

On the other hand, generalized continuum theories have been proposed to overcome the problem of the sizeindependent character exhibited by the classical theory. Within this category fall the works by Mindlin and Tiersten [2], Kröner [3], Toupin [4,5], Green and Rivlin [6], Mindlin [7,8] and Mindlin and Eshel [9], Yang et al. [10], Park and Gao [11,12], as well as the non-local continuum mechanics initiated by Eringen and coworkers [13-15], which has been widely used to analyse a variety of problems, such as wave propagation, dislocation, and crack singularities. In this respect, 1D discrete solids have served to provide physical foundation to different axiomatically formulated theories of generalized continuum [16,17], and to test their ability to mimic dispersive curves and to capture size effects [18].

Recently the models based on Fractional Calculus (FC), in which the differential operators are non-local and of an arbitrary (real or complex) order [19,20], have attracted increasing interest in different branches of Solid Mechanics. This mathematical approach has been introduced in describing the viscous behavior of materials, (cf. [21] and references therein), non-normal plastic flow [22], or finally spatial non-locality [23-29]. In the latter case, to the best of the authors' knowledge, the comparison with the Born-Von Karman model has not been studied before.

In this paper the dispersive effects in a 1D structured solid is analysed using the Fractional Continuum Mechanics (FCM) approach proposed by Sumelka [28,30], and Sumelka et al. [31]. This proposed formulation introduces non-locality in the spatial variable using Riesz-Caputo (RC) fractional derivative [32,33], and introduces two phenomenological/material parameters: 1) the order of fractional continua $\alpha$; and 2) the size of non-local surrounding $l_{f}$.

\footnotetext{
a e-mail: wojciech.sumelka@put.poznan.pl

b e-mail: ramon.zaera@uc3m.es

c e-mail: ppfer@ing.uc3m.es
} 


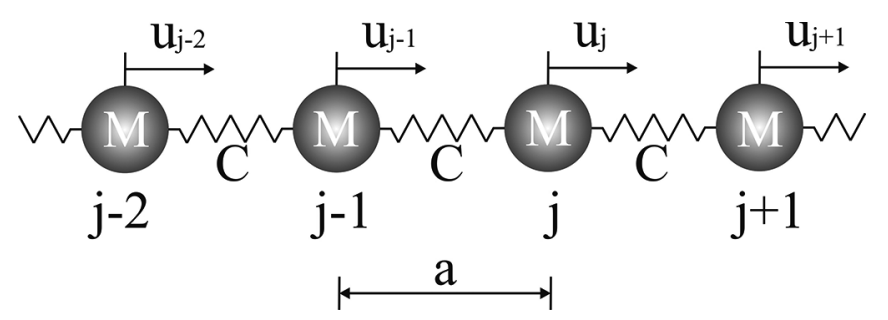

Fig. 1. Sketch of the Born-Von Karman (BK) lattice.

The analysis shows that $l_{f}$ plays the role of a distance between concentrated masses in the discrete model, while the parameter $\alpha$ can be used as a fitting parameter. The results reveal that with a suitable selection of the order of fractional differentiation $\alpha$, the dispersion curve of the Born-Von Karman model is well reproduced, proving the capability of the fractional model to capture the size effects present in the dynamic behaviour of discrete systems.

\section{Short review on the ability of selected continuum theories to capture the dispersive behavior of the Born-Von Karman lattice}

As a reference discrete model, we will consider a one-dimensional infinite chain of monoatomic particles at sites numbered $\ldots, j-1, j, j+1 \ldots$ with linear interactions between nearest neighbours, also called the Born-Von Karman (BK) lattice (cf. fig. 1). The mass of each particle is $M$, the stiffness of the interaction is $C$, and the lattice spacing at equilibrium is $a$. The position of the particle $j$ at the time $t$ is $x_{j}=j a+u_{j}$, where $j a=X_{j}$ is the position in the reference configuration and $u_{j}$ is the corresponding displacement. The equation of motion for the particle $j$ is given by

$$
M \ddot{u}_{j}=C\left(u_{j-1}-2 u_{j}+u_{j+1}\right) .
$$

The plane-wave solution for this equation is of the form

$$
u_{j}=U e^{i\left(\omega t-k X_{j}\right)},
$$

where $\omega$ is the angular frequency, $k$ is the wave number and $U$ is the wave amplitude. As $X_{j \pm q}=X_{j} \pm q a$, we have $u_{j \pm q}=u_{j} e^{ \pm i q k a}$, and eq. (1) permits to determine the dispersion relation for the lattice

$$
M \omega^{2}=2 C(1-\cos (k a)) .
$$

Defining dimensionless wave number $\bar{k}$ and frequency $\bar{\omega}$ as

$$
\bar{k}=k a, \quad \bar{\omega}=\frac{\omega a}{c_{0}},
$$

with $c_{0}^{2}=C a^{2} / M$, the non-dimensional dispersion relation also reads

$$
\bar{\omega}=2\left|\sin \left(\frac{\bar{k}}{2}\right)\right| .
$$

Due to its periodicity, this relation will be studied herein in the right half of the first Brillouin zone $0 \leq \bar{k} \leq \pi$. It is worth to emphasize that $\bar{\omega}$ is not a linear function of $\bar{k}$, therefore the BK lattice has a dispersive behaviour and the Fourier components of a signal will travel at different speeds. Moreover, in the short-wavelength limit $\bar{k}=\pi$, the phase and group velocity reach the following values:

$$
\left.\bar{\omega}\right|_{\bar{k}=\pi}=2,\left.\quad \frac{\mathrm{d} \bar{\omega}}{\mathrm{d} \bar{k}}\right|_{\bar{k}=\pi}=0,
$$

thus leading to a standing wave. In the long-wavelength limit $(\bar{k} \rightarrow 0)$, we get $\bar{\omega}=\bar{k}$. 


\subsection{Classic 1D continuum}

Special attention deserves the continuum limit of the lattice model [34], where the continuum displacement variable $u(X, t)$ fulfilling the conditions

$$
u\left(X_{j} \pm a, t\right)=u_{j \pm 1}(t)
$$

is assumed to vary slowly in the spatial coordinate, justifying the Taylor series expansion

$$
u\left(X_{j} \pm a, t\right) \approx u\left(X_{j}, t\right) \pm\left.\frac{\partial u}{\partial X}\right|_{X_{j}} a+\left.\frac{1}{2} \frac{\partial^{2} u}{\partial X^{2}}\right|_{X_{j}} a^{2} \pm \ldots
$$

Substituting in eq. (1), we get the classic linear 1D wave propagation equation

$$
\ddot{u}-c_{0}^{2} u^{\prime \prime}=0,
$$

where $(\ddot{\cdot})$ denotes the second-order derivative with respect to time, and $(\cdot)^{\prime \prime}$ denotes the second-order derivative with respect to spatial variable.

Defining the mass density $\rho_{0}$ and the Young modulus $E$ of an equivalent continuum media as

$$
\rho_{0}=\frac{M}{a^{3}}, \quad E=\frac{C}{a},
$$

we recover the wave speed in a $1 \mathrm{D}$ continuum

$$
c_{0}=\sqrt{\frac{E}{\rho_{0}}} .
$$

It is worth highlighting that the plane wave solution $u=U e^{i(\omega t-k X)}$ yields a non-dispersive relation between frequency and wave number $\bar{\omega}=\bar{k}$ (in nondimensional form), which is coincident with the long-wavelength limit of eq. (5).

\subsection{Strain gradient theories}

In the field of generalized continuum mechanics, several 1D models have been devised following the Mindlin's grade 2 (G2) and grade 3 (G3) strain gradient theories [35,36], including micro-inertia in the kinetic energy density [18], leading to the following motion equations, respectively:

$$
\begin{array}{ll}
\mathrm{G} 2: & \ddot{u}-\frac{h^{2}}{3} \ddot{u}^{\prime \prime}-c_{0}^{2}\left(u^{\prime \prime}-g^{2} u^{I V}\right)=0, \\
\mathrm{G} 3: & \ddot{u}-\frac{h^{2}}{3} \ddot{u}^{\prime \prime}-c_{0}^{2}\left[u^{\prime \prime}-\left(l_{11}^{2}+l_{12}^{2}\right) u^{I V}+l_{11}^{2} l_{12}^{2} u^{V I}\right]=0,
\end{array}
$$

where $h$ is a length scale parameter related to the micro-inertia, and $g, l_{11}, l_{12}$ are length scale parameters related to the strain energy potential.

Assuming a plane wave solution we obtain the following dispersion relations (in nondimensional form), respectively [18]:

$$
\begin{aligned}
& \text { G2 : } \quad \bar{\omega}=\bar{k} \sqrt{\frac{3\left(1+\bar{g}^{2} \bar{k}^{2}\right)}{3+\bar{h}^{2} \bar{k}^{2}}}, \\
& \text { G3 : } \bar{\omega}=\bar{k} \sqrt{\frac{3\left[1-\bar{c}_{1}^{2} \bar{k}^{2}+\bar{c}_{2}^{4} \bar{k}^{4}\right]}{3+\bar{h}^{2} \bar{k}^{2}}},
\end{aligned}
$$

with

$$
\bar{h}=h / a, \quad \bar{g}=g / a, \quad \bar{c}_{1}^{2}=-\frac{l_{11}^{2}+l_{12}^{2}}{a^{2}}, \quad \bar{c}_{2}^{4}=\frac{l_{11}^{2} l_{12}^{2}}{a^{4}} .
$$

The fulfillment of short-wavelength limit conditions (6) for the G2 model results in the following values for the lengthscale parameters [18]:

$$
\bar{g}^{2}=-\frac{4}{\pi^{4}}, \quad \bar{h}^{2}=\frac{3 \pi^{2}-24}{4 \pi^{2}}
$$


According to [18], both $\bar{h}$ and $\bar{g}$ should be positive real numbers for thermodynamic consistency, therefore this solution in unacceptable.

Regarding the G3 model, which retains three constants, fulfillment of conditions (6) plus the condition $\left.\bar{\omega}\right|_{\bar{k}=\pi / 2}=\sqrt{2}$ at the middle of the Brillouin zone results in the following two sets of values for the length scale parameters [18]:

$$
\left\{\bar{c}_{1}^{2}, \bar{c}_{2}^{4}, \bar{h}^{2}\right\}= \begin{cases}\left\{-\frac{16 \pi^{2}-164}{\pi^{4}},-\frac{8 \pi^{2}-80}{\pi^{6}}, \frac{9 \pi^{2}-88}{4 \pi^{2}}\right\}, & \text { (first set) } \\ \left\{\frac{5 \pi^{2}-32}{\pi^{4}}, \frac{4 \pi^{2}-32}{\pi^{6}},-\frac{1}{\pi^{2}}\right\}, & \text { (second set) } .\end{cases}
$$

According to [18] the second set is not an acceptable solution because it results in a kinetic energy density which is not positive definite (nor convex). On the other hand, the first set leads to an excellent fitting of the BK dispersion relation in the right half of the first Brillouin zone.

It is worth highlighting that thermodynamic inconsistencies found in the previous gradient theories are linked to the specific requirements imposed at the short-wavelength limit (and at the middle of the Brillouin zone for the G3 model). On the other side, alternative values of the length scale parameters fulfilling positive definiteness and convexity of both strain energy and kinetic energy potentials may lead to a mismatch in capturing the dispersive behaviour of the BK lattice.

\section{Dispersion in fractional continuum mechanics}

\subsection{Fractional $\omega$ vs. $k$ relation}

In the paper [31] the dynamic equilibrium for a 1D linear elastic body under the Sumelka Fractional Continuum Model (SFCM) was defined. Nevertheless, for the completeness of this paper let us once more recall some of the crucial aspects of this formulation. Thus, the governing equations for the 1D SFCM case can be stated as

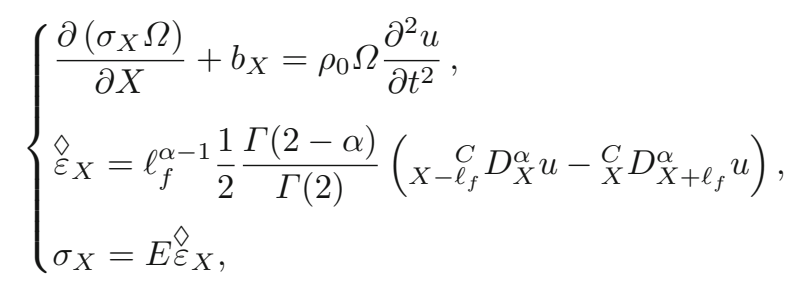

where $\sigma_{X}$ is the stress, $b_{X}$ is a body force, $\Omega$ is a cross section, $\hat{\varepsilon}_{X}$ is a fractional strain, $\Gamma$ is the Euler gamma function, $\ell_{f}$ and $\alpha$ are the length scale and order of SFCM, respectively, and ${ }_{a}^{C} D_{\chi}^{\alpha} f(\chi),{ }_{\chi}^{C} D_{b}^{\alpha} f(\chi)$ are the left and right Caputo's fractional derivatives defined as $[20,19,37]$

$$
\begin{aligned}
& { }_{a}^{C} D_{\chi}^{\alpha} f(\chi)=\frac{1}{\Gamma(n-\alpha)} \int_{a}^{\chi} \frac{f^{(n)}(\tau)}{(\chi-\tau)^{(\alpha-n+1)}} \mathrm{d} \tau \\
& { }_{\chi}^{C} D_{b}^{\alpha} f(\chi)=\frac{(-1)^{n}}{\Gamma(n-\alpha)} \int_{\chi}^{b} \frac{f^{(n)}(\tau)}{(\chi-\tau)^{(\alpha-n+1)}} \mathrm{d} \tau .
\end{aligned}
$$

Next, under the assumption of constant cross section $\left(\Omega=\right.$ const.) and omitting the body force term $\left(b_{X}=0\right)$ we obtain finally a single equation from eqs. (19) governing the 1D SFCM problem

$$
E \ell_{f}^{\alpha-1} \frac{1}{2} \frac{\Gamma(2-\alpha)}{\Gamma(2)} \frac{\partial}{\partial X}\left({ }_{X-\ell_{f}}^{C} D_{X}^{\alpha} u-{ }_{X}^{C} D_{X+\ell_{f}}^{\alpha} u\right)=\rho_{0} \frac{\partial^{2} u}{\partial t^{2}}
$$

In eqs. (20) and (21), $f^{(n)}$ is the $n$-th (integer) derivative of the function $f$ and $\alpha$ is in the range $(0,1][32,33]$.

Due to the fact that an analytical solution of eq. (22) is not available, the following logic, which assumes the analysis of the vibration of closed chain, has been applied to recover the $\omega$ vs. $k$ relation for SFCM. 

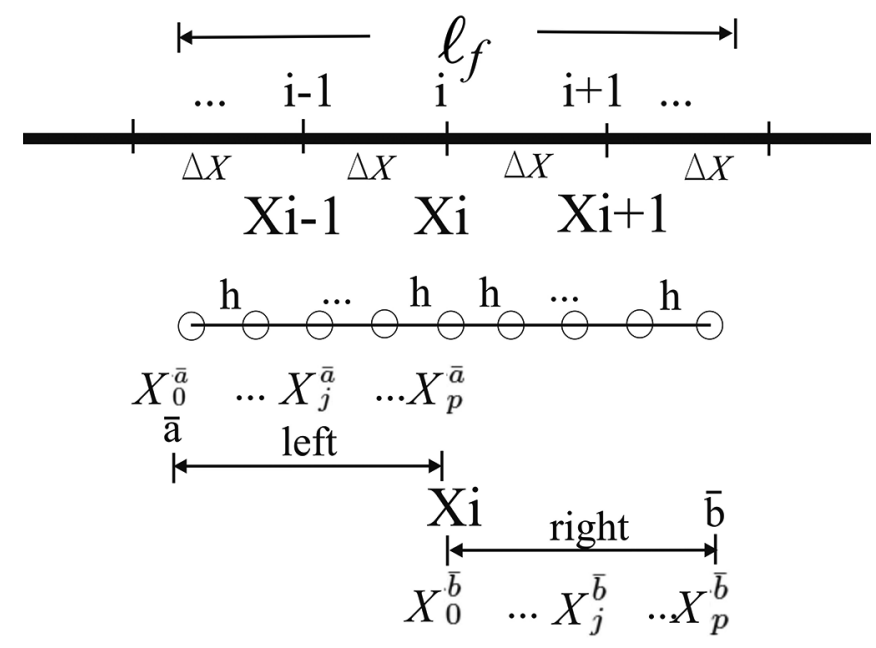

Fig. 2. Discretization of $1 \mathrm{D}$ fractional body.

The 1D domain of length $L$ was divided into $N$ intervals of equal length - thus, discrete points $X_{j}$ were located at the positions $X_{j}=j d$, where $j=0, \ldots, N$ and $N=\frac{L}{d}$. For each point $X_{j}$ the discrete counterpart of eq. (22) was obtained utilising the approximations of left and right Caputo's spatial derivatives (for fixed $t$ ) according to the expressions proposed in $[38,37]$, namely: i) for the left-sided derivatives we have

$$
\begin{aligned}
a & =X_{0}^{a}<X_{1}^{a}<\ldots<X_{j}^{a}<\ldots<X_{p}^{a}=X, \quad h=\frac{X_{p}^{a}-X_{0}^{a}}{p}=\frac{X-a}{p}, \quad p \geq 2, \\
X_{j}^{a}= & X_{0}^{a}+j h, \\
\left.{ }_{a}^{C} D_{X}^{\alpha} u(X)\right|_{x=x_{p}^{a}} \cong & \frac{h^{n-\alpha}}{\Gamma(n-\alpha+2)}\left\{\left[(p-1)^{n-\alpha+1}-(p-n+\alpha-1) p^{n-\alpha}\right] u^{(n)}\left(X_{0}^{a}\right)\right. \\
& \left.+u^{(n)}\left(X_{p}^{a}\right)+\sum_{j=1}^{p-1}\left[(p-j+1)^{n-\alpha+1}-2(p-j)^{n-\alpha+1}+(p-j-1)^{n-\alpha+1}\right] u^{(n)}\left(X_{j}^{a}\right)\right\}
\end{aligned}
$$

where $u^{(n)}\left(X_{j}^{a}\right)$ denotes the classical $n$-th derivative at $X=X_{j}^{a}$; ii) for the right-sided derivatives one obtains

$$
\begin{aligned}
& X=X_{0}^{b}<X_{1}^{b}<\ldots<X_{j}^{b}<\ldots<X_{p}^{b}=b, \quad h=\frac{X_{p}^{b}-X_{0}^{b}}{p}=\frac{b-X}{p}, \quad p \geq 2, \\
& X_{j}^{b}= X_{0}^{b}+j h \\
&\left.{ }_{X}^{C} D_{b}^{\alpha} u(X)\right|_{X=X_{0}^{b}} \cong(-1)^{n} \frac{h^{n-\alpha}}{\Gamma(n-\alpha+2)}\left\{\left[(p-1)^{n-\alpha+1}-(p-n+\alpha-1) p^{n-\alpha}\right] u^{(n)}\left(X_{p}^{b}\right)\right. \\
&\left.+u^{(n)}\left(X_{0}^{b}\right)+\sum_{j=1}^{p-1}\left[(j+1)^{n-\alpha+1}-2 j^{n-\alpha+1}+(j-1)^{n-\alpha+1}\right] u^{(n)}\left(X_{j}^{b}\right)\right\} .
\end{aligned}
$$

So, under the assumption that $\alpha \in(0,1) \rightarrow n=1$, the behaviour of the $i$-th node reads (cf. fig. 2)

$$
E \mathrm{~A}\left[\mathrm{~B} u_{0}^{a^{\prime \prime}}+\mathrm{C}^{a}(j) u_{j}^{a^{\prime \prime}}+u_{i}+\mathrm{C}^{b}(j) u_{j}^{b^{\prime \prime}}+\mathrm{B} u_{p}^{b^{\prime \prime}}\right]=\rho_{0} \frac{\partial^{2} u_{i}}{\partial t^{2}}
$$


where

$$
\begin{aligned}
\mathrm{A} & =\ell^{\alpha-1} \frac{\Gamma(2-\alpha)}{2} \frac{h^{1-\alpha}}{\Gamma(3-\alpha)}, \quad \mathrm{B}=\left[(p-1)^{2-\alpha}-(p-2+\alpha) p^{1-\alpha}\right], \\
\mathrm{C}^{a}(j) & =\left[(p-j+1)^{2-\alpha}-2(p-j)^{2-\alpha}+(p-j-1)^{2-\alpha}\right], \\
\mathrm{C}^{b}(j) & =\left[(j+1)^{2-\alpha}-2 j^{2-\alpha}+(j-1)^{2-\alpha}\right], \\
j & =1, \ldots, p-1 .
\end{aligned}
$$

Because on the considered subintervals $\left[X_{i-1}, X_{i}\right]$ it is assumed that $u$ varies linearly (cf. [37]), the points $u_{0}^{a}, u_{j}^{a}, u_{j}^{b}$ and $u_{p}^{b}$ can be independent of the discretization $\Delta X$ (cf. [31]). Thus, for independent parameters $h$ and $\Delta X$ the point $X_{j}^{a}$ influences two nodes in general, e.g. assuming that this point falls between the discretization points $X_{i-3}$ and $X_{i-2}$, we have

$$
u_{j}^{a}=u_{i-3}\left(1-\frac{X_{j}^{a}-X_{i-3}}{\Delta X}\right)+u_{i-2}\left(\frac{X_{j}^{a}-X_{i-3}}{\Delta X}\right) .
$$

The closed chain boundary conditions were assumed as mentioned, thus

$$
u_{0}=u_{N}
$$

and for virtual points outside the SFCM domain (cf. fig. 2)

$$
\begin{aligned}
u_{N+1} & =u_{1} ; \quad u_{N+2}=u_{2} ; \text { etc. }, \\
u_{-1} & =u_{N-1} ; \quad u_{-2}=u_{N-2} ; \text { etc. }
\end{aligned}
$$

On the other side the initial condition is

$$
u(t=0)=\sin \left(k_{m} X\right)
$$

where $k_{m}=\frac{2 \pi}{\lambda_{m}}=\frac{2 \pi m}{L}$ is the wave number and $m$ denotes the vibration mode number $(m=1,2, \ldots)$ - therefore the induced wavelength is $\lambda_{m}=\frac{L}{m}$.

In the next step, applying the Newmark method (average acceleration), the chain vibration was calculated to capture the displacement time-history of a selected control point (CP) (the analysis has shown that the influence of CP position is negligible). Finally, the Fast Fourier Transform (FFT) of the obtained CP displacement time-history was performed and the fundamental frequency $\omega$ was recovered; in other words, a point of the dispersion curve $\omega$ vs. $k$ was obtained. To minimise the leakage in the result of the FFT, the sine window filter (Hamming window) was also used [39-41].

\section{Analysis of results}

On the basis of the flow chart presented in the previous section we implemented the algorithm in a SCILAB environment and carried out computational simulations for different values of parameters $\ell_{f}[\mathrm{~m}]$ and $\alpha[-]$ assuming additionally that $E=1[\mathrm{~Pa}], \rho=1\left[\frac{\mathrm{kg}}{\mathrm{m}^{3}}\right], L=1[\mathrm{~m}]$, and $\Delta X=0.01[\mathrm{~m}]$. Recall that, based on SFCM definition, we have the restriction $L>2 \ell_{f}$, although the maximum value studied has been $\ell_{f}=0.15$ provided that the length scale parameters in non-local theories are usually not larger than $10 \%$ of the macroscopic characteristic dimension of the structure.

In fig. 3 the influence of parameters $\ell_{f}$ and $\alpha$ on the $\omega v s$. $k$ relation is presented. Both $\ell_{f}$ and $\alpha$ control the non-local effects, in the sense that $\alpha$ defines the way in which the information is governed from the region of influence, whereas $\ell_{f}$ describes the size of the non-local surrounding. It is noticeable that when $\ell_{f}$ is small the SFCM tends to a classical local formulation with a weak influence of $\alpha$.

The previous results derived with the fractional model can be contrasted with the dispersion relation of the BornVon Karman lattice. All results are normalised and plotted for the right half of the first Brillouin zone $0 \leq \bar{k} \leq \pi$ (fig. 4). It can be noticed that the length scale $\ell_{f}$ plays the role of the lattice spacing $a$, while the non-dimensional parameter $\alpha$ acts as a scaling parameter. Specifically, for an order of fractional derivative $\alpha \sim 0.55$, the model fulfils the limit condition $\left.\bar{\omega}\right|_{\bar{k}=\pi}=2$ and provides a close approximation to the dispersion of the BK lattice, even in the short-wavelength regime. As the value of $\alpha$ decreases, the difference between the frequencies of the discrete and the fractional models increases. 

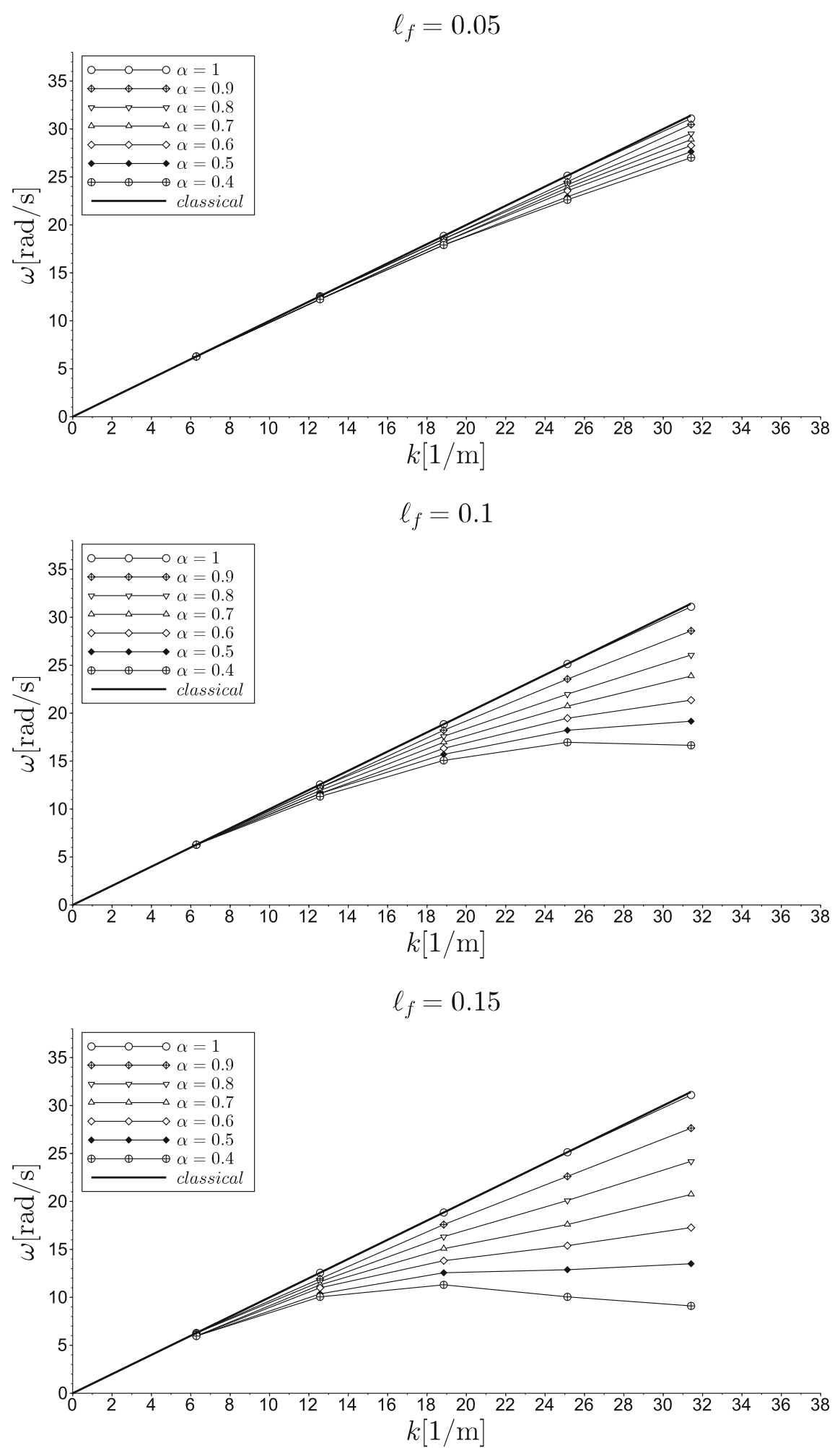

Fig. 3. $\omega$ vs. $k$ relation for different values of parameters $\ell_{f}$ and $\alpha$. 


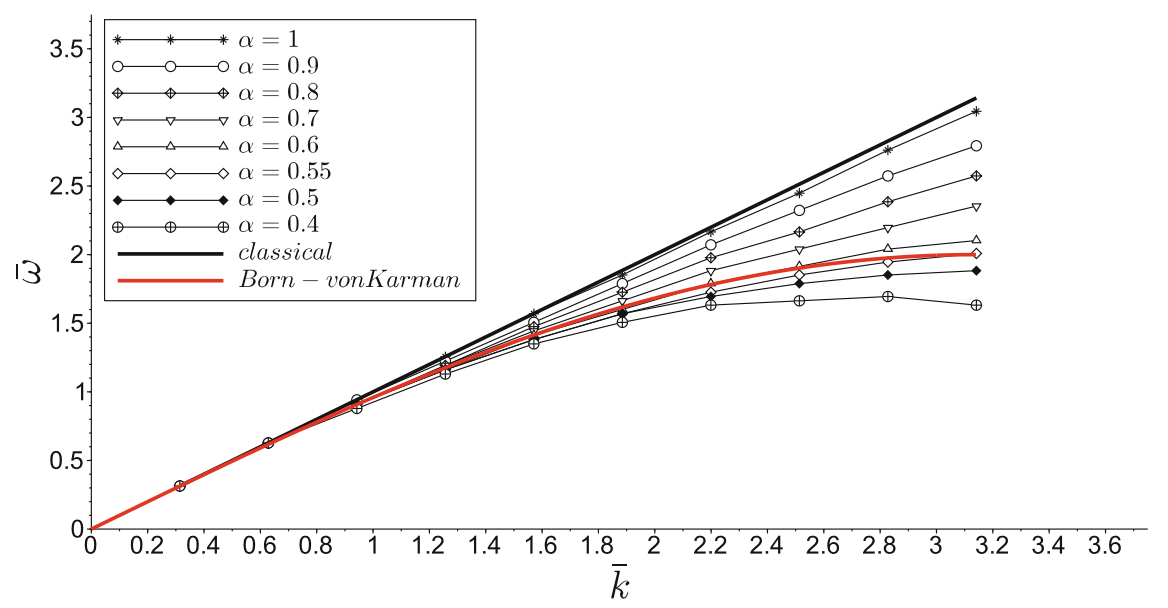

Fig. 4. SFCM vs. Born-Von Karman model.

\section{Conclusions}

This paper presents the analysis of the dispersive behaviour of a 1D solid undergoing axial vaibrations, using the Fractional Continuum Mechanics approach proposed by Sumelka [28,30]. The formulation uses the fractional RieszCaputo derivatives in the definition of the longitudinal deformation which, together with the Young modulus, leads to two additional model parameters: the size of the non-local surrounding $l_{f}$ and the order of the fraction derivative $\alpha$. A methodology for the direct time integration of the governing equations has been developed, and the vibratory behaviour of a cyclic 1D domain discretized as a chain of points has been compared to that of the Born-Von Karman lattice. The following key outcomes have been obtained:

- The model shows dispersive effects, evidencing the scale effects inherent to the approach of fractional calculus to Solid Mechanics.

- The dispersive features of the proposed model depend on the two parameters involved in the definition of the fractional derivative. The classical behaviour (non dispersive behaviour) is recovered for small values of the length scale parameter $l_{f}$, or for values of the derivative order $\alpha$ close to unit.

- A representation of the dispersion curves in nondimensional form permits to estabilish an equivalence between the scale parameter $l_{f}$ in the continuum fractional model and the lattice spacing $a$ in a discrete chain.

- The dispersion curve of the Born-Von Karman chain can be adequately reproduced with the fractional model, within the whole Brillouin zone.

All in all the work permitted to highlight, through the analysis of a 1D canonical problem, the ability of Fractional Continuum Mechanics to capture the dispersive nature of wave propagation phenomena in structured solids.

The authors wish to acknowledge the Ministerio de Economía y Competitividad de España for the financial support, under grant number DPI2014-57989-P.

Open Access This is an open access article distributed under the terms of the Creative Commons Attribution License (http://creativecommons.org/licenses/by/4.0), which permits unrestricted use, distribution, and reproduction in any medium, provided the original work is properly cited.

\section{References}

1. L. Brillouin, Wave propagation and group velocity (Academic Press, New York and London, 1960).

2. R.D. Mindlin, H.F. Tiersten, Arch. Ration. Mech. Anal. 11, 415 (1962).

3. E. Kröner, Int. J. Eng. Sci. 1, 261 (1963).

4. R.A. Toupin, Arch. Ration. Mech. Anal. 11, 385 (1963).

5. R.A. Toupin, Arch. Ration. Mech. Anal. 17, 85 (1964).

6. A.E. Green, R.S. Rivlin, Arch. Ration. Mech. Anal. 17, 113 (1964).

7. R.D. Mindlin, Arch. Ration. Mech. Anal. 16, 51 (1964). 
8. R.D. Mindlin, Int. J. Solids Struct. 1, 417 (1965).

9. R.D. Mindlin, N. Eshel, Int. J. Solids Struct. 4, 109 (1968).

10. F. Yang, A.C.M. Chong, D.C.C. Lam, P. Tong, Int. J. Solids Struct. 39, 2731 (2002).

11. S.K. Park, X.-L. Gao, J. Micromech. Microeng. 16, 2355 (2006).

12. S.K. Park, X.-L. Gao, Z. Angew. Math. Phys. 59, 904 (2008).

13. A.C. Eringen, Int. J. Eng. Sci. 10, 233 (1972).

14. A.C. Eringen, Int. J. Eng. Sci. 10, 1 (1972).

15. A.C. Eringen, J. Appl. Phys. 54, 4703 (1983).

16. D. Polyzos, D.I. Fotiadis, Int. J. Solids Struct. 49, 470 (2012).

17. V.E. Tarasov, Mod. Phys. Lett. B 28, 1450054 (2014).

18. D.A. Fafalis, S.P. Filopoulos, G.J. Tsamasphyros, Eur. J. Mech. A/Solids 36, 25 (2012).

19. A.A. Kilbas, H.M. Srivastava, J.J. Trujillo. Theory and Applications of Fractional Differential Equations (Elsevier, Amsterdam, 2006).

20. I. Podlubny, Fractional Differential Equations, in Mathematics in Science and Engineering, Vol. 198 (Academic Press, Cambridge, MA, 1999).

21. F. Mainardi, Fractional calculus and waves in linear viscoelasticity (Imperial College Press, London, 2010).

22. W. Sumelka, M. Nowak, Int. J. Numer. Anal. Methods Geomech. 40, 651 (2016).

23. M. Klimek, Czechoslov. J. Phys. 51, 1348 (2001).

24. K.A. Lazopoulos, Mech. Res. Communi. 33, 753 (2006).

25. T.M. Atanackovic, B. Stankovic, Acta Mech. 208, 1 (2009).

26. A. Carpinteri, P. Cornetti, A. Sapora, Eur. Phys. J. ST 193, 193 (2011).

27. C.S. Drapaca, S. Sivaloganathan, J. Elasticity 107, 107 (2012).

28. W. Sumelka, J. Therm. Stresses 37, 678 (2014).

29. K.A. Lazopoulos, A.K. Lazopoulos, Arch. Appl. Mech. 86, 1133 (2016).

30. W. Sumelka, T. Blaszczyk, C. Liebold, Eur. J. Mech. A/Solids 54, 243 (2015).

31. W. Sumelka, R. Zaera, J. Fernández-Sáez, Meccanica 50, 2309 (2015).

32. O.P. Agrawal, J. Phys. A 40, 6287 (2007).

33. G.S.F. Frederico, D.F.M. Torres, Appl. Math. Comput. 217, 1023 (2010).

34. G.A. Maugin, Nonlinear Waves in Elastic Crystals (Oxford University Press, New York, 1999).

35. R.D. Mindlin, Arch. Ration. Mech. Anal. 16, 51 (1964).

36. R.D. Mindlin, Int. J. Solids Struct. 1, 417 (1965).

37. J.S. Leszczyński, An introduction to fractional mechanics, Monographs No. 198 (The Publishing Office of Czestochowa University of Technology, 2011).

38. Z. Odibat, Appl. Math. Comput. 178, 527 (2006).

39. R.W. Ramirez, The FFT, Fundamentals and Concepts (Prentice-Hall, New Jersey, 1985).

40. W.M. Hartmann, Signals, Sound, and Sensation (AIP Press, Woodbury, NY, 1997).

41. R.E. Ziemer, W.H. Tranter, D.R. Fannin, Signals and Systems (Prentice Hall, New Jersey, 1998). 